\title{
Increasing Prevalence of Borrelia burgdorferi sensu stricto-Infected Blacklegged Ticks in Tennessee Valley, Tennessee, USA
}

\author{
Graham J. Hickling, Janetta R. Kelly, \\ Lisa D. Auckland, Sarah A. Hamer
}

In 2017, we surveyed forests in the upper Tennessee Valley, Tennessee, USA. We found Ixodes scapularis ticks established in 23 of 26 counties, 4 of which had Borrelia burgdorferi sensu stricto-infected ticks. Public health officials should be vigilant for increasing Lyme disease incidence in this region.

$\mathrm{I}$ $\mathrm{n}$ the United States, Lyme disease caused by tickborne bacterium Borrelia burgdorferi sensu stricto occurs primarily in the Northeast and upper Midwest (1). In eastern Tennessee, which is considered nonendemic for Lyme disease, most of the human population resides in a low-elevation swath of the Tennessee Valley bordered to the west by the Cumberland Plateau and the east by the Great Smoky Mountains. The vector of Lyme disease, the blacklegged tick Ixodes scapularis, was unreported in this area before 2006; in this year, uninfected adult ticks were collected from hunter-harvested deer in 8 Tennessee Valley counties (Figure 1, panel A) (2). This finding, plus uninfected I. scapularis ticks detected in Knox County in 2013, were later incorporated into the national distribution map for $I$. scapularis ticks (3).

During 2000-2014, human Lyme disease cases expanded southward along the eastern foothills of the Appalachian Mountains in nearby Virginia (4). In the winters of 2012 and 2013, B. burgdorferi-infected adult I. scapularis ticks were detected in Pulaski County, Virginia (5). This report of abundant infected I. scapularis ticks only 100 $\mathrm{km}$ from the Tennessee border motivated us to investigate whether Borrelia-infected ticks might now be present in the Tennessee Valley.

\section{The Study}

In late 2017, we sampled host-seeking I. scapularis ticks at 70 forested sites in 26 low-elevation counties in the upper Tennessee Valley (Figure 1, panel B). To find tick

Author affiliations: University of Tennessee Institute of Agriculture, Knoxville, Tennessee, USA (G.J. Hickling, J.R. Kelly); Texas A\&M University, College Station, Texas, USA (L.D. Auckland, S.A. Hamer)

DOI: https://doi.org/10.3201/eid2409.180343 habitats (hardwood or conifer forests $<800 \mathrm{~m}$ in elevation) accessible for sampling (i.e., trails through public forests or margins of public roads through private forests), we reviewed Google Earth (https://www.google. $\mathrm{com} /$ earth/) satellite imagery. We sampled each site once during the peak of adult I. scapularis tick activity (late October-January). We recorded site elevation and geocoordinates and collected host-seeking ticks using a standardized drag-cloth method; in brief, we dragged a $1-\mathrm{m}^{2}$ white corduroy cloth across leaf litter and checked every 10 paces for attached ticks. We dragged cloths 30-60 minutes per site and described tick tallies as number collected per hour to correct for variations in effort per site. We did not conduct drag-cloth collections during periods of rain, strong wind, low air temperatures $\left(<8^{\circ} \mathrm{C}\right)$, or low relative humidity $(<40 \%)$.

We placed ticks in $70 \%$ ethanol, identified species using a morphologic key (6), and tested ticks for Borrelia spirochete infection by DNA extraction and quantitative multiplex real-time PCR using differential probes targeting the 16S rDNA of Lyme group Borrelia and relapsing fever group Borrelia (7). We then subjected a random subset of negative samples and samples positive by the $16 \mathrm{~S}$ assay (maximum 6 samples/site) to PCR amplification of the 16S-23S rDNA intergenic spacer region (8) and Sanger sequencing for species-level identification.

No previous tick drag-cloth counts existed for the counties in our survey area, except for a 1,050-m transect of land in a forest in Anderson County, which we have drag-cloth sampled annually each December since 2012. To assess a trend in adult $I$. scapularis tick abundance, we applied linear regression modeling to the tick tallies from that transect of land.

In late 2017, we collected 479 adult $I$. scapularis ticks from 49 of 70 sites in the upper Tennessee Valley. Two adult Amblyomma americanum ticks collected during the survey were excluded from analysis. We detected I. scapularis ticks in all 26 counties surveyed, 23 of which met the criterion used by Eisen et al. for established I. scapularis populations (Figure 1, panel B) (3). Site elevations were 210-730 m; the highest elevation at which I. scapularis ticks were found was $570 \mathrm{~m}$. The average number of adult ticks collected per hour during drag-cloth surveys was 8.8 (range 0-48). At the Anderson County site that had been 

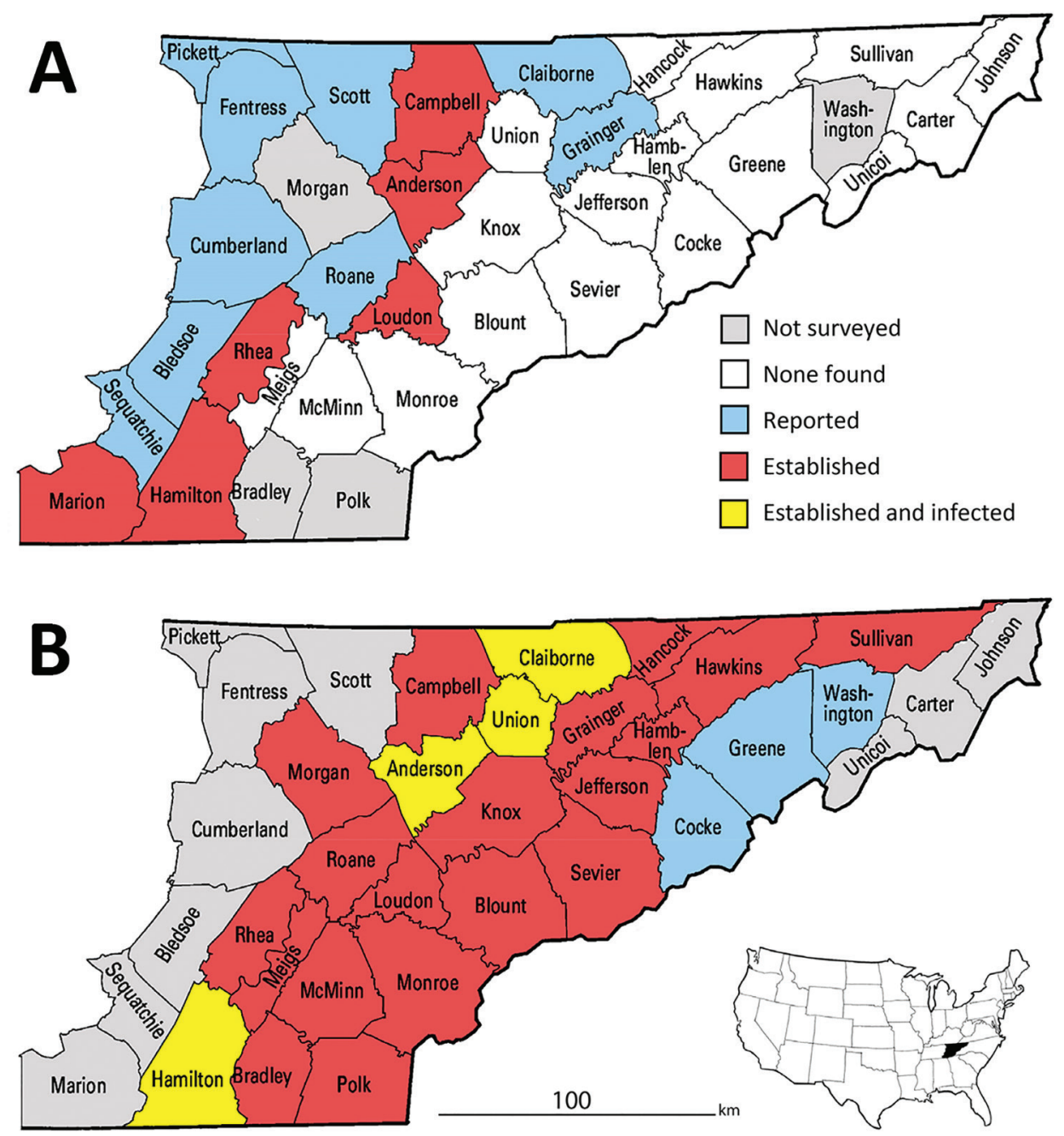

Figure 1. County-level distribution of Ixodes scapularis ticks and Borrelia burgdorferiinfected $I$. scapularis ticks in upper Tennessee Valley, USA, 2006 and 2017. A county was classified as having an established $I$. scapularis population if $\geq 6 \mathrm{l}$. scapularis adult ticks or ticks of 2 life stages were collected in that county. A county was classified as having $I$. scapularis ticks reported if $1-5 \mathrm{I}$. scapularis ticks of a single life stage were collected in that county. A county was classified as infected if $I$. scapularis ticks infected with $B$. burgdorferi were detected in that county. A) I. scapularis ticks in 2006 (2), determined by collecting ticks from hunterharvested deer. B) I. scapularis ticks in 2017 determined by drag-cloth surveying during the peak of adult tick activity (late October-January). drag-cloth sampled annually, a highly significant increasing trend in I. scapularis ticks was evident ( $\mathrm{p}=0.003$; Figure 2); the count in 2017 (24.8 ticks/hour) was $3.5 \times$ higher than that in 2012.

We tested all I. scapularis ticks collected $(\mathrm{N}=$ 479) for Borrelia spp. infection; 46 ticks (9.6\%) from 7 sites in 4 counties (Anderson, Claiborne, Hamilton, and Union; Figure 1, panel B) tested positive for Lyme group Borrelia by $16 \mathrm{~S}$ rDNA PCR screening. We tested 26 samples for the intergenic spacer region by PCR; all were positive for this sequence and identified as B. burgdorferi sensu stricto by sequencing. Most infected ticks came from 2 Union County sites, which had prevalences of $44 \%(14 / 32)$ and $78 \%(18 / 23)$. No ticks were found to be infected with B. miyamotoi or other relapsing fever group borreliae.

\section{Conclusions}

In eastern Tennessee, public awareness and concern about ticks focuses primarily on the abundant lone star ticks
(Amblyomma americanum) and American dog ticks (Dermacentor variabilis) encountered during the spring and summer. Both species can spread pathogens (9), but neither are vectors of $B$. burgdorferi spirochetes. Immature I. scapularis ticks are similarly active in the summer, but in southern states, these ticks typically avoid host-seeking above leaf litter and are rarely seen on humans or dragcloths (10). For this reason, assessment of I. scapularis distribution in southern states is best achieved by acquiring adult life-stage ticks during cool season drag-cloth surveys (as reported here) or by collecting ticks from deer harvested in the fall. Inspection of hunter-harvested deer is efficient for the detection of low-density $I$. scapularis ticks (11). Thus, our drag-cloth sampling for I. scapularis ticks in 14 counties where none were found on deer a decade ago (Figure 1, panels A, B) suggests that tick abundance in these counties has increased. This suggestion is supported by a $>3$-fold increase in I. scapularis tick counts at the Anderson County site where we have 6 consecutive years of drag-cloth counts. 


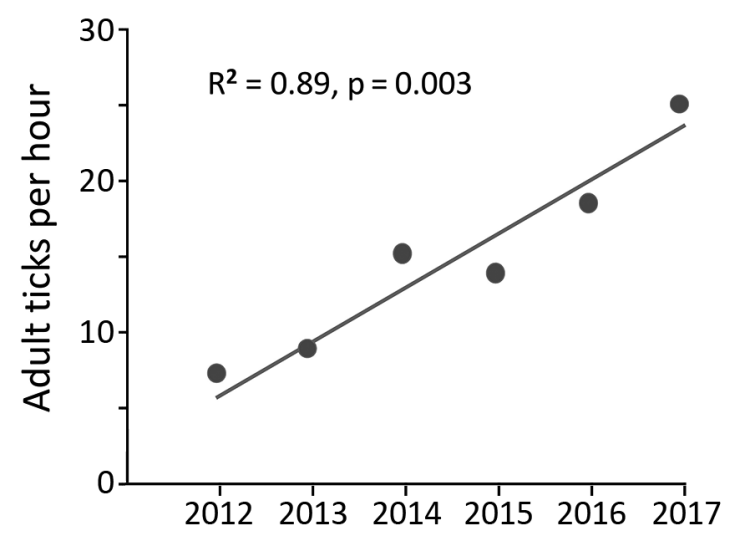

Figure 2. Six-year trend in adult Ixodes scapularis tick counts at Forest Resources Research and Education Center $\left(36.00^{\circ} \mathrm{N}\right.$, $84.22^{\circ} \mathrm{W}$; elevation $298 \mathrm{~m}$ ), Anderson County, Tennessee, USA, 2012-2017. We collected host-seeking $I$. scapularis adult ticks by drag-cloth sampling vegetation on a 1,050-m transect of mixed hardwood forest once each December.

This study documents emergence of B. burgdorferi senso stricto in tick populations in eastern Tennessee. Infected ticks were predominantly found in high-prevalence hot spots in Union County $\left(36.39^{\circ} \mathrm{N}\right)$. Relative to Lyme disease-endemic areas in the north, B. burgdorferi prevalence in the study area was low $(10 \%)$ and had a patchy distribution (7/49 sites had positive ticks). This distribution could reflect host barriers of $B$. burgdorferi transmission in the South (12), or more concerning, the hot spots in Union County might reflect the beginning of an infection surge, similar to that seen in southwestern Virginia during the past decade (4).

In the United States, Lyme disease is primarily a summertime disease associated with bites from nymphal I. scapularis ticks. In southern states, detection of $B$. burgdorferi bacteria in adult ticks does not necessarily imply risk to humans; for example, $B$. burgdorferi cycles in I. scapularis populations on the Outer Banks of North Carolina, yet nymphs in that area cannot be collected on drag-cloths and no locally acquired cases of Lyme disease have been reported (13). In contrast, infected nymphs have been found on drag-cloths from surveys in Virginia, where Lyme disease incidence has spiked (14). We speculate that Borrelia-infected I. scapularis populations emerging in southwestern Virginia include immigrant ticks from the North, with some nymphs in these populations exhibiting host-seeking behaviors that lead to contact with humans. A similar invasion process might be under way in eastern Tennessee; the surveillance data reported here provide a baseline for investigating this possibility. Health officials and practitioners need to be vigilant for increasing Lyme disease incidence in Tennessee.

\section{Acknowledgments}

We thank the University of Tennessee's Forest Resources AgResearch and Education Center staff and land owners and managers for access to survey sites. Tyler Noll and James Hickling assisted with field collections.

This work was supported by the US Department of Agriculture National Institute of Food and Agriculture Hatch project 1012932 (to G.J.H.).

\section{About the Author}

Dr. Hickling is professor in the Center for Wildlife Health at the University of Tennessee, Knoxville, Tennessee, USA. His research focuses on the eco-epidemiology of tickborne diseases in the southeastern United States.

\section{References}

1. Mead PS. Epidemiology of Lyme disease. Infect Dis Clin North Am. 2015;29:187-210. http://dx.doi.org/10.1016/ j.idc.2015.02.010

2. Rosen ME, Hamer SA, Gerhardt RR, Jones CJ, Muller LI, Scott MC, et al. Borrelia burgdorferi not detected in widespread Ixodes scapularis (Acari: Ixodidae) collected from white-tailed deer in Tennessee. J Med Entomol. 2012;49:1473-80. http://dx.doi.org/10.1603/ME11255

3. Eisen RJ, Eisen L, Beard CB. County-scale distribution of Ixodes scapularis and Ixodes pacificus (Acari: Ixodidae) in the continental United States. J Med Entomol. 2016;53:349-86. http://dx.doi.org/10.1093/jme/tjv237

4. Lantos PM, Nigrovic LE, Auwaerter PG, Fowler VG Jr, Ruffin F, Brinkerhoff RJ, et al. Geographic expansion of Lyme disease in the southeastern United States, 2000-2014. Open Forum Infect Dis. 2015;2:ofv143. http://dx.doi.org/10.1093/ ofid/ofv143

5. Herrin BH, Zajac AM, Little SE. Confirmation of Borrelia burgdorferi sensu stricto and Anaplasma phagocytophilum in Ixodes scapularis, southwestern Virginia. Vector Borne Zoonotic Dis. 2014;14:821-3. http://dx.doi.org/10.1089/ vbz.2014.1661

6. Keirans JE, Clifford CM. The genus Ixodes in the United States: a scanning electron microscope study and key to the adults. J Med Entomol Suppl. 1978;2:1-149.

7. Tsao JI, Wootton JT, Bunikis J, Luna MG, Fish D, Barbour AG. An ecological approach to preventing human infection: vaccinating wild mouse reservoirs intervenes in the Lyme disease cycle. Proc Natl Acad Sci U S A. 2004;101:18159-64. http://dx.doi.org/10.1073/pnas.0405763102

8. Bunikis J, Garpmo U, Tsao J, Berglund J, Fish D, Barbour AG. Sequence typing reveals extensive strain diversity of the Lyme borreliosis agents Borrelia burgdorferi in North America and Borrelia afzelii in Europe. Microbiology. 2004;150:1741-55. http://dx.doi.org/10.1099/mic.0.26944-0

9. Stromdahl EY, Hickling GJ. Beyond Lyme: aetiology of tick-borne human diseases with emphasis on the south-eastern United States. Zoonoses Public Health. 2012;59(Suppl 2):48-64. http://dx.doi.org/10.1111/j.1863-2378.2012.01475.x

10. Arsnoe IM, Hickling GJ, Ginsberg HS, McElreath R, Tsao JI. Different populations of blacklegged tick nymphs exhibit differences in questing behavior that have implications for human Lyme disease risk. PLoS One. 2015;10:e0127450. http://dx.doi.org/10.1371/journal.pone.0127450 
11. Lockwood BH, Stasiak I, Pfaff MA, Cleveland CA, Yabsley MJ. Widespread distribution of ticks and selected tick-borne pathogens in Kentucky (USA). Ticks Tick Borne Dis. 2018;9:738-41. http://dx.doi.org/10.1016/j.ttbdis.2018.02.016

12. Apperson CS, Levine JF, Evans TL, Braswell A, Heller J. Relative utilization of reptiles and rodents as hosts by immature Ixodes scapularis (Acari: Ixodidae) in the coastal plain of North Carolina, USA. Exp Appl Acarol. 1993;17:719-31.

13. Levine JF, Apperson CS, Levin M, Kelly TR, Kakumanu ML, Ponnusamy L, et al. Stable transmission of Borrelia burgdorferi sensu stricto on the Outer Banks of North Carolina. Zoonoses
Public Health. 2017;64:337-54. http://dx.doi.org/10.1111/ zph.12302

14. Brinkerhoff RJ, Gilliam WF, Gaines D. Lyme disease, Virginia, USA, 2000-2011. Emerg Infect Dis. 2014;20:1661-8. http://dx.doi.org/10.3201/eid2010.130782

Address for correspondence: Graham J. Hickling, University of Tennessee Institute of Agriculture, Center for Wildlife Health, 274 Ellington Bldg, 2431 Joe Johnson Dr, Knoxville, TN 37996, USA; email: ghicklin@utk.edu

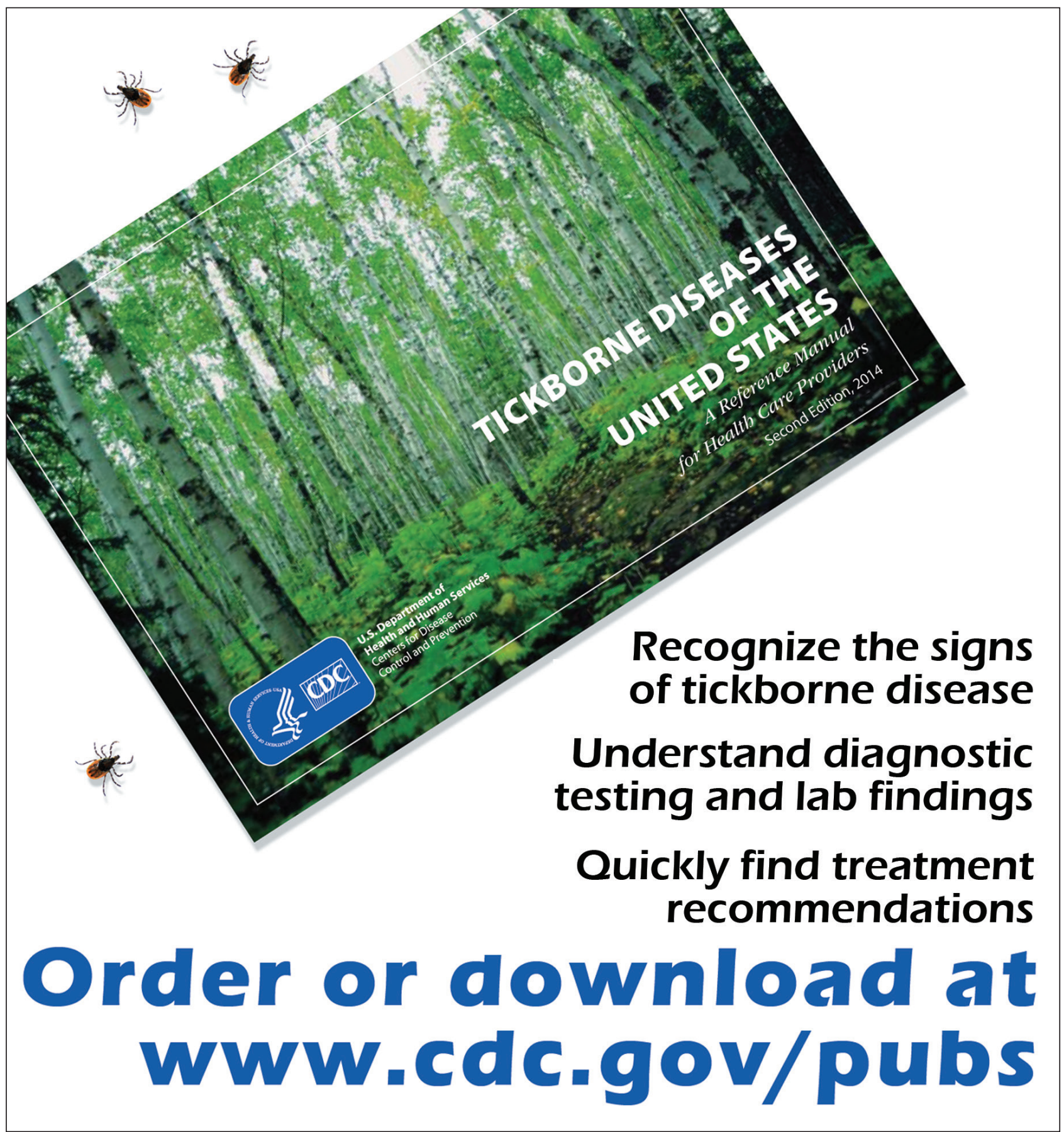

